\title{
Unraveling the CHIP:Hsp70 complex as an information processor for protein quality control
}

\author{
Jamie VanPelt and Richard C. Page* \\ Department of Chemistry and Biochemistry, Miami University, Oxford, Ohio 45056, USA \\ *Correspondence: pagerc@miamioh.edu (R.C.P.)
}

Short title: CHIP:Hsp70 Protein Quality Control

\section{Keywords}

- protein quality control

- ubiquitination

- protein refolding

- allostery

- dynamics

\section{Page 1}




\section{Summary}

The CHIP:Hsp70 complex stands at the crossroads of the cellular protein quality control system. Hsp70 facilitates active refolding of misfolded client proteins, while CHIP directs ubiquitination of misfolded client proteins bound to Hsp70. The direct competition between CHIP and Hsp70 for the fate of misfolded proteins leads to the question: how does the CHIP:Hsp70 complex execute triage decisions that direct misfolded proteins for either refolding or degradation? The current body of literature points toward action of the CHIP:Hsp70 complex as an information processor that takes inputs in the form of client folding state, dynamics, and posttranslational modifications, then outputs either refolded or ubiquitinated client proteins. Herein we examine the CHIP:Hsp70 complex beginning with the structure and function of CHIP and Hsp70, followed by an examination of recent studies of the interactions and dynamics of the CHIP:Hsp70 complex. 


\section{INTRODUCTION}

Protein quality control is essential for maintaining cellular homeostasis [1]. The accumulation of misfolded proteins can lead to cell death and various diseases [2]. In order to maintain proper folding of proteins, a complex group of molecular machines work together to determine the fate of misfolded proteins [3]. Chaperone proteins, such as heat shock protein 70 (Hsp70), recruit misfolded protein "clients" and simultaneously attempt client refolding while inhibiting client aggregation [4]. Concurrently, the E3 ubiquitin ligase CHIP (carboxy-terminus of $\underline{H s p 70}$ interacting protein) binds to the Cterminal domain of Hsp70 [5-8], essentially hijacking the recruiting efforts of Hsp70 in an effort to place CHIP in proximity to misfolded proteins [9]. These combined activities of CHIP and Hsp70 place the CHIP:Hsp70 complex at the crossroads of degradative and refolding pathways in eukaryotes $[1,3,10]$. The CHIP:Hsp70 complex performs chaperoned ubiquitination, a process that ubiquitinates misfolded proteins recruited to Hsp70 [3,10-14]. Clients of the CHIP:Hsp70 complex that are targets for ubiquitination include epidermal growth factor receptor [15], androgen receptor [16], estrogen receptor- $\alpha$ [17], hypoxia-inducible factor HIF-1 $\alpha$ [18], the inducible and neuronal nitric oxide synthases iNOS and nNOS [8,19], and the cystic fibrosis transmembrane conductance regulator $[20,21]$. The selection of targets by the CHIP:Hsp70 complex appears to utilize recognition or detection of folding states or folding intermediates by Hsp70 $[10,21,22]$ as a common thread that recruits misfolded clients to the CHIP:Hsp70 complex. In fact, a recent study identified several client-binding modes by Hsp70 that enable recruitment of unfolded, partially folded, and near-native proteins [23]. This, in coordination with binding of CHIP to the carboxy-terminal domains of Hsp70 [6-8], Page 3 
combines to position CHIP and generate productive encounter complexes that ubiquitinate Hsp70-bound misfolded clients $[8,10,12]$. Unfortunately, the full complement of factors that determine the fate of an Hsp70-bound client within the CHIP:Hsp70 chaperoned ubiquitination complex are not fully known. However, the function of the CHIP:Hsp70 complex and its ability to choose between two opposing pathways [12,24], refolding or degradation, suggest that the complex acts much like an information processor. The ability for the CHIP:Hsp70 complex to make triage decisions $[12,14,24]$ raises the question: what inputs are utilized by the CHIP:Hsp70 complex in making triage decisions? Structural, biochemical, and biophysical studies of CHIP and Hsp70 suggest that dynamics, allostery, and posttranslational modifications may play key roles, however future studies are required to determine the range of inputs and their role in the triage and decision making process. To facilitate these studies, this review seeks to present the status of the field with an eye toward directions that require further exploration. Beginning with details regarding the structure and function of Hsp70, the review shifts to discuss key features of CHIP, followed by an analysis of current knowledge of the CHIP:Hsp70 interaction. The protein quality control field is ripe for breakthroughs and the CHIP:Hsp70 interaction represents an important target for development of therapeutics and for our understanding of the underlying biochemistry and biophysics.

\section{Hsp70 and the recruitment of misfolded client substrates}

\subsection{Diversity and conformity of heat shock proteins as chaperones}

Generally categorized into one of five families [25], heat shock proteins serve vital, often complementary roles in stabilizing or refolding misfolded proteins. The HSPA (HSP70) Page 4 
and HSPH (HSP110) family is comprised of large, often oligomeric species [26] with monomer molecular weights of approximately $70 \mathrm{kDa}(\mathrm{HSPA})$ and $110 \mathrm{kDa}(\mathrm{HSPH})$, respectively. The DNAJ (HSP40) family is a large group of proteins encoded by fifty different genes that fall into one of three subfamilies, $A, B$, or $C$ based upon organization of domains and location of the J-domain [25]. The ubiquitous J-domain confers HSPA-binding to the DNAJ family and serves to stimulate nucleotide hydrolysis [27], though the structure of the HSPA/DNAJ complex and mechanism of activation are under debate $[28,29]$. The HSPB family of small heat shock proteins is distinguished by the crystallin domain and includes the well-studied $\alpha A$ crystallin (HSBP4) and $\alpha B$ crystallin (HSBP5) members. The highly oligomeric $\alpha$-crystallins comprise nearly $30 \%$ of the protein content within the lens of the eye and play a key role in maintaining lens protein solubility [30]. A recent study identified $\alpha$-crystallins as viable drug targets for the treatment of cataracts [31]. The HSPC (HSP90) family is also found, at times, in complex with HSPA proteins, together functioning to refold misfolded complexes [32]. A recent cryoelectron microscopy structure provided a glimpse at the structural organization of HSPA/HSPC complexes facilitated by the tetratricopeptide repeat (TPR) motif-containing Hsp70/Hsp90 organizing protein (HOP) [33]. In comparison, members of the HSPD/E family function as complexes of HSPD/E proteins that exist in isolation as a single, large, roughly cylindrical oligomer [34]. The HSPD/E family are homologs of the Escherichia coli GroEL (Hsp60) and GroES (Hsp10) proteins, with both mitochondrial and cytosolic (TRiC/CCT) family members [25,34].

2.2 Allosteric modulation and structural changes along the client binding cycle HSP70 family chaperones contain two structural domains, the nucleotide binding Page 5 
domain (NBD) responsible for ATPase activity and a substrate-binding domain (SBD) that binds client proteins for refolding. The SBD is divided into a $\beta$-sheet subdomain ( $\beta$ SBD) and the $\alpha$-helical lid subdomain (Fig. 1) that work together to form a clamp that binds client proteins. The SBD and NBD are joined by a leucine-rich interdomain linker, which has been thought to be involved in the regulation of substrate binding kinetics [35-37]. Much of the structural knowledge for the remarkable structural changes (Fig. 1) that occur across the Hsp70 client refolding cycle has come from studies using DnaK, the Escherichia coli homolog of Hsp70. The mechanism of the client refolding cycle (Fig. 2) begins with an Hsp70 chaperone in the ATP-bound state. A structure of ATPbound DnaK, with mutations introduced to prevent ATP hydrolysis while stabilizing ATP association [38], identified an open structure with the helical lid subdomain unbound from the $\beta$-SBD and substantial interactions between the $\beta$-SBD and NBD (Fig. 1 and 2). In this conformation, the substrate-binding domain has weak millimolar affinity for the misfolded client protein [39]. Subsequently, a J-domain co-chaperone, such as Hsp40 or DnaJ, induces ATP hydrolysis by the Hsp70-NBD resulting in a conformational change in Hsp70 that promotes binding of client proteins at the SBD [40]. Although a recent paper uncovered additional, alternative client-binding modes for partially-folded or near-native clients [23], recruitment of misfolded clients to the SBD is viewed as the canonical mechanism for binding to misfolded clients $[22,39,41]$. An NMR study of DnaK in the ADP-bound state, along with a model NRLLLTG peptide client, found that the helical lid subdomain clamps across the client peptide [42]. The clamping action holds the client in place on the $\beta$-SBD while the entire SBD becomes uncoupled from the NBD 
[42]. Clamping of the helical lid subdomain onto the $\beta$-SBD locks the client protein in place and dramatically increases affinity for the client to approximately $300 \mathrm{nM}$ [40]. Several hypotheses including Brownian ratchet [43] and power stroke [44] mechanisms have been proposed to drive refolding after client binding, however an entropic pulling model was recently posited that more fully recapitulates Hsp70 functions [45]. The increased dynamics observed upon uncoupling of the SBD from the NBD [42] lend additional credence to the entropic pulling mechanism [45] and decrease likelihood of the power stroke model. After binding the client a nucleotide exchange factor (NEF) binds to the Hsp70-NBD triggering release of ADP. Dissociation of the NEF returns Hsp70 to the nucleotide free state, which through allostery, results in a conformational change of the SBD and client release. Repeated cycles of nucleotide binding and hydrolysis, client binding, nucleotide dissociation and client release enable active refolding of a misfolded client [46]. Despite our knowledge of the Hsp70 client refolding mechanism, significant questions remain. For example, how do cells triage clients that are not successfully refolded? Is there a time limit on how long Hsp70 can attempt refolding for any single client?

Recently, the interdomain linker between the NBD and SBD, which participates in allosteric modulation of $\mathrm{Hsp70}[35,37]$, was implicated in facilitating Hsp70 oligomerization [26]. Studies suggest the formation of both well ordered dimers $[47,48]$ and heterogeneous oligomers are mediated by the SBD [49] or the NBD-SBD interdomain linker [26], indicating that the mechanism of oligomerization is not yet settled within the community. Many years prior to the recent wave of structural data it was suggested that the oligomeric state of Hsp70 may play a role in regulating its Page 7 
function [50]. In fact, the formation and purpose of Hsp70 oligomers has been reported to depend on temperature [50], and either regulate protein refolding activity by locking Hsp70 into a non-active oligomeric complex [49], or facilitate interactions with other proteins including Hsp40 [48] and Hsp90 [47]. Another study reported that oligomerization of $\mathrm{Hsp} 70$ is thought to potentially hold a reservoir of Hsp70 protomers in a stabilized state with reduced functionality that, once presented with a misfolded substrate, triggers release of functional monomers that can initiate protein refolding [26]. The reports of different effects of multiple oligomerization modes on activity indicates that additional studies are needed to more conclusively determine the mechanism and purpose of oligomerization. In particular, the effect of Hsp70 oligomerization upon binding to CHIP has not been reported.

\section{CHIP, an E3 ubiquitin ligase with dynamic motions}

\subsection{Structural diversity in CHIP}

E3 ubiquitin ligases are traditionally classified into three families, HECT, U-box, and RING, though hybrid RING-between-RING (RBR) ligases [51] were recently identified. The HECT and RBR families are differentiated by their ability to form covalent intermediates with ubiquitin prior to covalent modification of the client. In contrast, the $U$ box and RING family facilitate direct transfer of ubiquitin from the E2 to the client by positioning the substrate in close proximity to the E2-ubiquitin thioester (Fig. 3). CHIP is a U-box E3 ubiquitin ligase with three discrete domains, the N-terminal tetratricopeptide repeat (TPR) domain, the C-terminal U-box domain, and the intermediate helical linker (Fig. 4a). TPR domains are a ubiquitous structural motif of three to sixteen repeats of a 34 residue conserved sequence [52]. Each TPR repeat produces a helix-turn-helix Page 8 
architecture that contributes to a superhelical fold with distinct ligand binding grooves suitable for peptide binding or scaffolding [52-57]. The CHIP-TPR domain features a peptide binding cleft formed by three consecutive TPR motifs flanked by a seventh helix that extends toward the linker domain $[6,8]$. Like homologous TPR domains, CHIP-TPR recognizes the IEEVD motif at the C-terminus of chaperones such as Hsp70 and HSP90 at low micromolar affinity [58]. A recent study also identified additional interactions between the CHIP-TPR and the helical lid subdomain of Hsp70 [8] reminiscent of the interactions between TPR domains and globular domains observed in structures of Ppp5 phosphatase in complex with the Ppp5 TPR [59] and the structure of p67 $7^{\text {phox }}$ in complex with Rac [60].

While the TPR domain facilitates binding to chaperones, the U-box domain of CHIP confers ubiquitin ligase activity by recruiting E2 ubiquitin conjugating enzymes. Structures of CHIP-U-box in complex with UbcH5 and Ubc13 $[6,61]$ have been solved and are similar to structures of U-box or RING-domain E3 ligases in complex with E2 ubiquitin conjugating enzymes [62,63]. Interestingly, CHIP shows surprisingly little selectivity in recruitment of E2 ubiquitin conjugating enzymes and has been shown to catalyze ubiquitin transfer when paired with any of eight E2 enzymes from the UbcH5ac, UbcH6a-c, Ube2W, and Ubc13 families [64,65]. The U-box domain is structurally similar to RING domains found in many E3 ubiquitin ligases, with the key exception that structural zinc atoms in RING domains are replaced by a hydrogen bond network in the U-box [66,67]. The structural similarity with RING-type E3 ubiquitin ligases allows researchers studying CHIP to capitalize on recent structures of RING-E3 enzymes in complex with E2 ubiquitin conjugates [68-71] to produce models (Fig. 4b) of CHIP in 
complex with an E2 ubiquitin conjugate [8]. Importantly these models are fully consistent with simultaneous binding of CHIP to an E2 ubiquitin conjugate via the CHIP-U-box and to Hsp70 via the CHIP-TPR [8].

Centrally located between the U-box and TPR domains the helical linker within CHIP not only bridges the U-box and TPR domains, it is also a key contributor to CHIP dimerization [6] and appears to be a source of substantial conformational variation $[6,61,72]$. Crystal structures of CHIP exhibit two distinct conformations for the helical linker with an asymmetric dimer (Fig. 5a) conformation featuring a kinked linker helix in one structure [6] and a symmetric dimer (Fig. 5b) with a pair of straight linker helices in the second structure [61]. The arrangement of each CHIP protomer across the two structures is of particular interest and has led to studies probing the flexibility and dynamics of CHIP [72,73], including a study that identified flexibility as a key requirement for CHIP E3 ligase activity [73]. Additionally, the asymmetric arrangement of CHIP protomers occludes one of the E2 binding sites and allows for only a single ubiquitination-competent complex to form per dimer [6] while the symmetric arrangement is capable of binding two E2 enzymes simultaneously. Thus, asymmetric/symmetric switching would offer a potential mode of regulation in which overall structure of CHIP controls the E3 ubiquitin ligase activity [6].

\subsection{Dynamics within CHIP}

While the existing crystal structures of CHIP provide valuable snapshots and key details on dimerization and interactions with binding partners, these structures do not provide the full picture of dynamics and motions that are inherent within CHIP in solution $[72,73]$. A recent computational modeling study found that the CHIP homodimer could 
switch between the asymmetric and symmetric form [73]. In this dynamic model, CHIP moves between both symmetric and asymmetric conformations without disrupting dimerization sites. This dynamic model alludes to the complex dynamics that allow CHIP to facilitate ubiquitin transfer to a wide range of client proteins. As CHIP is responsible for ubiquitination of more than just a single class of proteins [24], client proteins will likely vary in structure, size, and location of target lysine residues. Although it is still unknown how specific lysine residues from a client protein are targeted for ubiquitin transfer from CHIP-bound E2 ubiquitin conjugates, biochemical and computational studies suggest that the flexibility of CHIP may play a key role $[72,73]$.

The binding of key protein partners, such as Hsp70 or E2 enzymes, is also known to alter the flexibility and dynamics of CHIP [72]. HDX-MS measurements of amide hydrogen exchange rates in the presence and absence of Hsp70 and E2 enzymes [72] provide a proxy for dynamics and suggest that, in the absence of binding partners, CHIP is highly dynamic and flexible. Reduced dynamics upon Hsp70 binding are particularly notable in the CHIP-TPR domain which exhibits reductions in deuterium incorporation rates of one to two orders of magnitude upon binding to Hsp70 [72]. Anecdotally, the high flexibility of the TPR when not bound to Hsp70 suggests that the absence of a crystal structure for free CHIP-TPR is not for want of trying, rather the flexible CHIP-TPR may present a high entropic barrier to crystallization. The linker region also exhibits 10 -fold higher amide hydrogen exchange rates in the absence of Hsp70 due to its flexible nature, while the U-box domain was more protected as evidenced by HD exchange rates differing by less than 2-fold between free and Hsp70bound states [72]. Regions of CHIP involved in dimerization, as identified in the 
asymmetric and symmetric crystal structures, were confirmed by the HDX-MS data. Interestingly the HDX-MS data does not appear to favor either the symmetric or asymmetric dimer structures of CHIP. It is unclear as to whether the lack of clear preference between the two structures is due to stable populations of both structures, or a time averaged fluctuation between alternating conformations of the asymmetric dimer that pass through the symmetric dimer state in transition.

\section{Interactions between CHIP and Hsp70}

\subsection{TPR-IEEVD interactions and modulations in disease}

In order to facilitate polyubiquitination of a bound client protein, Hsp70 binds to the TPR domain of CHIP. The CHIP-TPR is located at the N-terminus and consists of seven $\alpha-$ helices arranged as three pairs of antiparallel $\alpha$-helices, while the seventh helix is elongated leading into the helical linker domain and is packed against one of the helical pairs (Fig. 6a). Chaperone proteins bind to the electropositive TPR domain via a strongly electronegative C-terminal EEVD motif [6]. The Hsp70 C-terminal EEVD motif sequence "GSGPTIEEVD" differs from the Hsp90 EEVD motif sequence "DDTSRMEEVD" in the residues preceding the "EEVD" sequence. Although the EEVD motif facilitates the essential interactions with the TPR domain, CHIP can accommodate different chaperones in the hydrophobic binding pocket of the TPR domain due to variations in conformation (Fig. 6b) of bound EEVD motif containing peptides [6,8,74]. While a majority of co-chaperones, including CHIP, have been shown to bind to chaperones as long as they contain the EEVD motif [58], selectivity appears to be gained by residues preceding the "EEVD" sequence, ruling out the EEVD motif as the 
sole source for regulating chaperone binding. In fact, the EEVD motif binding cleft formed by CHIP-TPR appears to be optimized for binding to the Hsp70-PTIEEVD motif, exhibiting a $K_{d}$ values of 0.4 or $0.6 \mu \mathrm{M}[7,58]$ compared to $K_{d}$ values of 1.0 to $4.9 \mu \mathrm{M}$ for CHIP-TPR binding to the Hsp90 "SRMEEVD" motif [58,75]. In particular, the lle/Met immediately preceding the "EEVD" motif appears to confer the majority of the difference in binding affinities between Hsp70 and Hsp90 to CHIP [58].

Posttranslational modifications likely play a key role in determining whether a client protein will be refolded or degraded as these posttranslational modifications result in differential binding of co-chaperones to Hsp70 and HSP90 [75]. The key posttranslational modification for chaperone regulation is phosphorylation of a serine or threonine near the EEVD motif. These serine and threonine residues, highly conserved among eukaryotic HSP70 and HSP90 families, provide phosphorylation sites for various kinases, including CK1, CK2, and GSK3 $\beta$ [75]. The phosphorylation state of the Cterminus of Hsp70 and Hsp90 regulates the interaction of these chaperones with the TPR domain of two co-chaperones, CHIP and HOP [75]. Interestingly, phosphorylation of Hsp70 "PTIEEVD" and Hsp90 "SRMEEVD" motifs results in only a 2-fold reduction in affinity from $4.9 \mu \mathrm{M}$ to $9 \mu \mathrm{M}$ for binding to Hsp90 compared to a 10 -fold reduction in affinity from $1.9 \mu \mathrm{M}$ to $20 \mu \mathrm{M}$ for binding to Hsp70 [75]. This differential effect of binding affinity is consistent with existing structures of Hsp70 and Hsp90 "EEVD" motifs bound to CHIP-TPR (Fig. 6c). For Hsp90, the position and orientation of the Hsp90 "SRMEEVD" motif serine would likely result in only minor changes to binding upon phosphorylation. In contrast, the position of the Hsp70 "PTIEEVD" motif threonine (Fig. 6b) indicates that phosphorylation of this threonine could result in steric clashes with the Page 13 
CHIP-TPR that would pose a significant detriment to binding [8]. Despite the analyses made possible by existing data, structures of phosphorylated Hsp70 and Hsp90 "EEVD" motifs in complex with CHIP-TPR would enable a more complete analysis of the scope of mechanisms that control chaperone binding and for defining how post-translational modifications act as an additional source of selectivity for interactions between chaperones and co-chaperones [75].

In contrast to the 10 -fold reductions in binding affinity for phosphorylated Hsp70 to CHIP and 2-fold reduction for phosphorylated Hsp90 binding to CHIP, affinity of phosphorylated Hsp70 for HOP remains nearly constant at approximately $4 \mu \mathrm{M}$ to $6 \mu \mathrm{M}$ and affinity for phosphorylated Hsp90 improves from $6 \mu \mathrm{M}$ to $3 \mu \mathrm{M}$ [75]. Thus, the nonphosphorylated C-termini of Hsp70 and HSP90 preferentially bind to the TPR domain of CHIP in order to subject chaperone bound client proteins to ubiquitination while phosphorylated Hsp70 and Hsp90 preferentially bind to HOP, which promotes client protein refolding $[33,75]$. Interestingly, the regulation of chaperone function through phosphorylation has been shown to play a role in human cancer cells [75]. Previous work demonstrated that cancer cells take advantage of chaperone activity, and targeting chaperones for inhibition has been studied as potential cancer treatments [76]. Although an early study posited that cancer cells disrupt co-chaperone activity by decreasing expression of CHIP [77], more recent work has shown contradicting results [75]. It has now been shown that CHIP expression levels are not altered in cancer cells; rather HOP expression is greatly increased along with greater levels of Hsp70 and Hsp90 phosphorylation. Chaperone phosphorylation paired with overexpression of HOP enables cancer cells to effectively direct and regulate co-chaperone binding to promote Page 14 
protein refolding over degradation. These results were seen in melanoma, bladder, breast, lung, and pancreatic cancer cell lines, suggesting that cancer cells regulate cochaperone binding as a mechanism to diminish degradation and escalate refolding of client proteins [75]. Cancer cells promote protein folding rather than degradation due to the need for robust cell proliferation in order to survive. Since cell proliferation requires an excess of protein levels [78], cancer cells prevent protein degradation in order to promote cancer cell replication [75].

\subsection{Additional interactions beyond the Hsp70 IEEVD peptide}

The most well studied interaction between CHIP and Hsp70 is the binding of the Hsp70 EEVD motif to the CHIP-TPR. Upon further investigation of the CHIP:Hsp70 interaction, a novel transient binding site was observed [7]. Biolayer interferometry measurements of the difference in binding affinity between full length Hsp70 and a truncated Hsp70 construct containing the PTIEEVD motif showed that full length Hsp70 bound CHIP with a 6-fold tighter affinity when compared to the EEVD peptide. These findings suggested that Hsp70 interacts with CHIP in a previously unobserved and transient manner [7]. To further investigate CHIP:Hsp70 binding, TROSY NMR studies were performed. Spectra of full-length Hsp70 compared to the CHIP:Hsp70 complex yielded very little changes, except in the EEVD motif, further suggesting that intermolecular interactions outside the EEVD motif would be transient [7]. NMR relaxation experiments support that Hsp70 is tethered to the CHIP-TPR domain, but the remaining domains move freely of each other, much like "beads on a string". The mobility of the CHIP:Hsp70 complex likely allows for flexible movements that can accommodate myriad conformations in order for CHIP to locate the lysine residues of Hsp70 bound client proteins for targeting Page 15 
ubiquitination [7]. However, the view of the CHIP:Hsp70 complex as "beads on a string" does not fully recapitulate the observed binding data demonstrating an increase in affinity due to Hsp70 elements outside the EEVD motif. Additionally, the "beads on a string" model does not explain the pattern of CHIP-meditated ubiquitination of $\mathrm{Hsc70/Hsp70} \mathrm{that} \mathrm{is} \mathrm{biased} \mathrm{toward} \mathrm{lysine} \mathrm{residues} \mathrm{near} \mathrm{the} \mathrm{SBD} \mathrm{and} \mathrm{C-terminus} \mathrm{[79].}$

Our recent studies have shown that the co-chaperone to chaperone interaction is much more complex than previously thought [8]. This structural data identified an additional site of interaction between CHIP and Hsc70 (Fig. 6c). Through a series of HSQC-NMR titrations with CHIP and an Hsc70-lid-tail mutant that lacks the C-terminal GPTIEEVD motif, extensive line broadening was observed. These results indicated that an interaction between CHIP and Hsc70 occurs outside of the EEVD motif in either the lid subdomain or the tail region. To further identify the nature of these novel interactions, a crystal structure of CHIP-TPR with an Hsc70-lid-tail-EEVD construct was determined (Fig. 6c). The $2.91 \AA$ structure featured well-defined regions of the EEVD motif and the Hsc70-lid, however, the electron density for much of the Hsc70-tail domain was highly disordered [8]. As expected, based on previous studies [74], the PTIEEVD motif of Hsc70 was shown to interact with the TPR domain. The new structure allows for fulllength CHIP:Hsp70 complexes to be modeled (Fig. 7a-b). These complexes are compatible with simultaneous interaction of CHIP with Hsp70 and an E2 ubiquitin conjugate, and provide a glimpse (Fig. 7c) of the organization of chaperoned ubiquitination cascades [8]. This structural data, which suggests that the CHIP:Hsp70 complex can adopt different conformations based on the nucleotide binding status of Hsp70 (Fig. 7), raises questions about how the CHIP:Hsp70 complex bridges the gap 
between the E2 ubiquitin active site and lysine residues on an Hsp70-bound client. Although additional studies are needed to answer these questions, flexibility or dynamics of CHIP [72,73] and Hsp70 [80] likely play a role [8].

Interestingly, the structure also revealed new insights into the interactions between the Hsc70-lid and CHIP. The Hsc70-lid interacts with CHIP through the side chains from Cterminal helix of the Hsc70-lid and the beginning residues of the tail region. The sites of interaction in the TPR domain include the N-terminal region of helix 1, and the loops between helices 2-3, 4-5, and 6-7 [8]. The overall structure suggests that the Hsc70-lid caps one side of the CHIP-TPR domain. The majority of the tail domain, except for the PTIEEVD motif residues that interact with the TPR domain, remain disordered. Functionally, these novel interactions were found to be responsible for regulating CHIPmeditated ubiquitination of $\mathrm{Hsc70}$ and $\mathrm{Hsp} 70 / \mathrm{Hsc70}$ clients. Mutations to CHIP in the sites that interact with the Hsc70-lid abolished Hsc70 and client ubiquitination. The Hsc70-lid:CHIP-TPR interaction has a predicted binding affinity of less than $100 \mu \mathrm{M}$, consistent with the previous CHIP:Hsp70 interaction study [7], suggesting that Hsc70lid:CHIP-TPR interaction exists intermittently while the Hsc70 EEVD motif remains tethered to the CHIP-TPR domain [8]. One possible explanation for the importance of this weak interaction is to allow CHIP to dock and undock from the Hsc70-lid in order to assist in targeting an Hsc70-bound client lysine residue for ubiquitination.

\section{Perspective}

The CHIP:Hsp70 complex exhibits the hallmarks of an information processor. Data taken as inputs likely include, but may not be limited to CHIP:Hsp70 complex populations, unfolded client binding, dynamics within CHIP, Hsp70, and client proteins, Page 17 
along with posttranslational modifications. These inputs are then translated into directed ubiquitination of CHIP, termed autoubiquitination, Hsp70, or the misfolded client [14]. The bulk of the field has focused primarily studies of Hsp70 $[42,81,82]$ or CHIP $[72,73]$ and has begun a shift toward studying CHIP:Hsp70 complexes in more detail $[7,8]$. With the valuable insights these studies have provided, the stage is set for in-depth explorations of the dynamic structures of CHIP:Hsp70:client complexes. Future studies will elucidate the role that each type of input plays in determining the output, or target and level of ubiquitination. These studies will likely need to draw upon combinations of expertise from the structural, biophysical, and biochemical realms to determine the mechanisms that regulate the CHIP:Hsp70 in its role linking protein refolding and degradation in protein quality control. With mechanistic studies on the horizon that will link the biophysics and biochemistry of the CHIP:Hsp70 complex to the outcomes of in vivo protein quality control, the future of the CHIP:Hsp70 field is bright and primed for significant advances.

\section{Conclusion}

The CHIP:Hsp70 complex brings together two divergent arms of cellular protein quality control. The delicate balance between refolding and degradation can be shifted by small changes in binding affinity, in the range of 2 to $10 \mu \mathrm{M}$, that promote or diminish CHIP:Hsp70 complex formation. Inputs in the form of dynamics and posttranslational modifications have demonstrated effects on the output of the CHIP:Hsp70 complex by modulating ubiquitination levels and targets. Responsiveness of the CHIP:Hsp70 information processor is maintained by bipartite binding via strong interactions, on the order of $0.5 \mu \mathrm{M}$, between the Hsp70 EEVD motif and CHIP-TPR domain which serve to Page 18 
tether CHIP to Hsp70, paired with transient interactions, with less than $100 \mu \mathrm{M}$ affinity, of the Hsp70-lid to the CHIP-TPR domain which position CHIP with respect to Hsp70 and clients. The output of the CHIP:Hsp70:client complex is directed ubiquitination of one or more components. In this way, the CHIP-Hsp70 complex functions as an information processor, translating dynamic cellular inputs into functional outcomes of the cellular protein quality control system.

\section{Acknowledgments}

The authors acknowledge financial support from the US National Science Foundation (Award No. MCB 1552113 to RCP), the American Heart Association (Award No. 16SDG26960000 to RCP), the Burroughs Wellcome Fund (Award No. 1014031 to $\mathrm{RCP}$ ), and institutional support from Miami University through the Robert $\mathrm{H}$. and Nancy J. Blayney Professorship (to RCP).

\section{REFERENCES}

[1] B. Chen, M. Retzlaff, T. Roos, J. Frydman, Cellular strategies of protein quality control, Cold Spring Harb Perspect Biol. 3 (2011) a004374-a004374. doi:10.1101/cshperspect.a004374.

[2] F. Trcka, B. Vojtesek, P. Muller, Protein quality control and cancerogenesis, Klin Onkol. 25 Suppl 2 (2012) 2S38-44.

[3] D.M. Cyr, J. Höhfeld, C. Patterson, Protein quality control: U-box-containing E3 ubiquitin ligases join the fold, Trends Biochem. Sci. 27 (2002) 368-375. doi:10.1016/S0968-0004(02)02125-4.

[4] M.P. Mayer, B. Bukau, Hsp70 chaperones: cellular functions and molecular mechanism, Cell. Mol. Life Sci. 62 (2005) 670-684. doi:10.1007/s00018-0044464-6.

[5] C.A. Ballinger, P. Connell, Y. Wu, Z. Hu, L.J. Thompson, L.Y. Yin, et al., Identification of CHIP, a novel tetratricopeptide repeat-containing protein that interacts with heat shock proteins and negatively regulates chaperone functions, 
Mol Cell Biol. 19 (1999) 4535-4545.

[6] M. Zhang, M. Windheim, S.M. Roe, M. Peggie, P. Cohen, C. Prodromou, et al., Chaperoned ubiquitylation--crystal structures of the CHIP U box E3 ubiquitin ligase and a CHIP-Ubc13-Uev1a complex, Mol. Cell. 20 (2005) 525-538. doi:10.1016/j.molcel.2005.09.023.

[7] M.C. Smith, K.M. Scaglione, V.A. Assimon, S. Patury, A.D. Thompson, C.A. Dickey, et al., The E3 ubiquitin ligase CHIP and the molecular chaperone Hsc70 form a dynamic, tethered complex, Biochemistry. 52 (2013) 5354-5364. doi:10.1021/bi4009209.

[8] H. Zhang, J. Amick, R. Chakravarti, S. Santarriaga, S. Schlanger, C. McGlone, et al., A Bipartite Interaction between Hsp70 and CHIP Regulates Ubiquitination of Chaperoned Client Proteins, Structure. 23 (2015) 472-482. doi:10.1016/j.str.2015.01.003.

[9] S. Murata, Y. Minami, M. Minami, T. Chiba, K. Tanaka, CHIP is a chaperonedependent E3 ligase that ubiquitylates unfolded protein, EMBO Rep. 2 (2001) 1133-1138. doi:10.1093/embo-reports/kve246.

[10] P. Connell, C.A. Ballinger, J. Jiang, Y. Wu, L.J. Thompson, J. Hohfeld, et al., The co-chaperone CHIP regulates protein triage decisions mediated by heatshock proteins, Nat. Cell Biol. 3 (2001) 93-96. doi:10.1038/35050618.

[11] J. Demand, S. Alberti, C. Patterson, J. Hohfeld, Cooperation of a ubiquitin domain protein and an E3 ubiquitin ligase during chaperone/proteasome coupling, Curr. Biol. 11 (2001) 1569-1577.

[12] M. Stankiewicz, R. Nikolay, V. Rybin, M.P. Mayer, CHIP participates in protein triage decisions by preferentially ubiquitinating Hsp70-bound substrates, Febs J. 277 (2010) 3353-3367. doi:10.1111/j.1742-4658.2010.07737.x.

[13] S. Lee, D.W. Lee, Y. Lee, U. Mayer, Y.-D. Stierhof, S. Lee, et al., Heat shock protein cognate 70-4 and an E3 ubiquitin ligase, CHIP, mediate plastid-destined precursor degradation through the ubiquitin-26S proteasome system in Arabidopsis, Plant Cell. 21 (2009) 3984-4001. doi:10.1105/tpc.109.071548.

[14] S.-B. Qian, H. McDonough, F. Boellmann, D.M. Cyr, C. Patterson, CHIPmediated stress recovery by sequential ubiquitination of substrates and Hsp70, Nature. 440 (2006) 551-555. doi:10.1038/nature04600.

[15] T. Wang, J. Yang, J. Xu, J. Li, Z. Cao, L. Zhou, et al., CHIP is a novel tumor suppressor in pancreatic cancer through targeting EGFR, Oncotarget. 5 (2014) 1969-1986. doi:10.18632/oncotarget.1890.

[16] S. Sarkar, D.L. Brautigan, S.J. Parsons, J.M. Larner, Androgen receptor degradation by the E3 ligase CHIP modulates mitotic arrest in prostate cancer cells, Oncogene. 33 (2014) 26-33. doi:10.1038/onc.2012.561.

[17] M. Fan, A. Park, K.P. Nephew, CHIP (carboxyl terminus of Hsc70-interacting protein) promotes basal and geldanamycin-induced degradation of estrogen receptor-alpha, Mol. Endocrinol. 19 (2005) 2901-2914. doi:10.1210/me.20050111.

[18] W. Luo, J. Zhong, R. Chang, H. Hu, A. Pandey, G.L. Semenza, Hsp70 and CHIP selectively mediate ubiquitination and degradation of hypoxia-inducible factor (HIF)-1alpha but Not HIF-2alpha, J. Biol. Chem. 285 (2010) 3651-3663. 
doi:10.1074/jbc.M109.068577.

[19] H.-M. Peng, Y. Morishima, G.J. Jenkins, A.Y. Dunbar, M. Lau, C. Patterson, et al., Ubiquitylation of neuronal nitric-oxide synthase by CHIP, a chaperonedependent E3 ligase, J. Biol. Chem. 279 (2004) 52970-52977. doi:10.1074/jbc.M406926200.

[20] G.C. Meacham, C. Patterson, W. Zhang, J.M. Younger, D.M. Cyr, The Hsc70 co-chaperone CHIP targets immature CFTR for proteasomal degradation, Nat. Cell Biol. 3 (2001) 100-105. doi:10.1038/35050509.

[21] J.M. Younger, H.Y. Ren, L. Chen, C.-Y. Fan, A. Fields, C. Patterson, et al., A foldable CFTR $\triangle$ F508 biogenic intermediate accumulates upon inhibition of the Hsc70-CHIP E3 ubiquitin ligase, The Journal of Cell Biology. 167 (2004) 10751085. doi:10.1083/jcb.200410065.

[22] R. Schlecht, A.H. Erbse, B. Bukau, M.P. Mayer, Mechanics of Hsp70 chaperones enables differential interaction with client proteins, Nat. Struct. Mol. Biol. 18 (2011) 345-351. doi:10.1038/nsmb.2006.

[23] A. Mashaghi, S. Bezrukavnikov, D.P. Minde, A.S. Wentink, R. Kityk, B. Zachmann-Brand, et al., Alternative modes of client binding enable functional plasticity of Hsp70, Nature. (2016). doi:10.1038/nature20137.

[24] H. McDonough, C. Patterson, CHIP: a link between the chaperone and proteasome systems, Cell Stress Chaperones. 8 (2003) 303-308.

[25] H.H. Kampinga, J. Hageman, M.J. Vos, H. Kubota, R.M. Tanguay, E.A. Bruford, et al., Guidelines for the nomenclature of the human heat shock proteins, Cell Stress Chaperones. 14 (2009) 105-111. doi:10.1007/s12192-008-0068-7.

[26] F.A. Aprile, A. Dhulesia, F. Stengel, C. Roodveldt, J.L.P. Benesch, P. Tortora, et al., Hsp70 oligomerization is mediated by an interaction between the interdomain linker and the substrate-binding domain, PLoS ONE. 8 (2013) e67961. doi:10.1371/journal.pone.0067961.

[27] F. Hennessy, W.S. Nicoll, R. Zimmermann, M.E. Cheetham, G.L. Blatch, Not all $\mathrm{J}$ domains are created equal: implications for the specificity of Hsp40-Hsp70 interactions, Protein Sci. 14 (2005) 1697-1709. doi:10.1110/ps.051406805.

[28] A. Ahmad, A. Bhattacharya, R.A. McDonald, M. Cordes, B. Ellington, E.B. Bertelsen, et al., Heat shock protein $70 \mathrm{kDa}$ chaperone/DnaJ cochaperone complex employs an unusual dynamic interface, Proc Natl Acad Sci USA. 108 (2011) 18966-18971. doi:10.1073/pnas.1111220108.

[29] J. Jiang, E.G. Maes, A.B. Taylor, L. Wang, A.P. Hinck, E.M. Lafer, et al., Structural basis of $\mathrm{J}$ cochaperone binding and regulation of Hsp70, Mol. Cell. 28 (2007) 422-433. doi:10.1016/j.molcel.2007.08.022.

[30] M. Haslbeck, T. Franzmann, D. Weinfurtner, J. Buchner, Some like it hot: the structure and function of small heat-shock proteins, Nat. Struct. Mol. Biol. 12 (2005) 842-846. doi:10.1038/nsmb993.

[31] L.N. Makley, K.A. McMenimen, B.T. DeVree, J.W. Goldman, B.N. McGlasson, P. Rajagopal, et al., Pharmacological chaperone for a-crystallin partially restores transparency in cataract models, Science. 350 (2015) 674-677. doi:10.1126/science.aac9145.

[32] R. Zhao, W.A. Houry, Hsp90: a chaperone for protein folding and gene 
regulation, Biochem. Cell Biol. 83 (2005) 703-710. doi:10.1139/005-158.

[33] S. Alvira, J. Cuéllar, A. Röhl, S. Yamamoto, H. Itoh, C. Alfonso, et al., Structural characterization of the substrate transfer mechanism in Hsp70/Hsp90 folding machinery mediated by Hop, Nat. Commun. 5 (2014) 5484.

doi:10.1038/ncomms6484.

[34] M.A. Kabir, W. Uddin, A. Narayanan, P.K. Reddy, M.A. Jairajpuri, F. Sherman, et al., Functional Subunits of Eukaryotic Chaperonin CCT/TRiC in Protein Folding, J Amino Acids. 2011 (2011) 843206-16. doi:10.4061/2011/843206.

[35] M. Vogel, M.P. Mayer, B. Bukau, Allosteric regulation of Hsp70 chaperones involves a conserved interdomain linker, J. Biol. Chem. 281 (2006) 3870538711. doi:10.1074/jbc.M609020200.

[36] J.F. Swain, E.G. Schulz, L.M. Gierasch, Direct comparison of a stable isolated Hsp70 substrate-binding domain in the empty and substrate-bound states, J. Biol. Chem. 281 (2006) 1605-1611. doi:10.1074/jbc.M509356200.

[37] J.F. Swain, G. Dinler, R. Sivendran, D.L. Montgomery, M. Stotz, L.M. Gierasch, Hsp70 Chaperone Ligands Control Domain Association via an Allosteric Mechanism Mediated by the Interdomain Linker, Mol. Cell. 26 (2007) 27-39. doi:10.1016/j.molcel.2007.02.020.

[38] R. Qi, E.B. Sarbeng, Q. Liu, K.Q. Le, X. Xu, H. Xu, et al., Allosteric opening of the polypeptide-binding site when an Hsp70 binds ATP, Nat. Struct. Mol. Biol. 20 (2013) 900-907. doi:10.1038/nsmb.2583.

[39] M.P. Mayer, Gymnastics of molecular chaperones, Mol. Cell. 39 (2010) 321331. doi:10.1016/j.molcel.2010.07.012.

[40] R. Kityk, M. Vogel, R. Schlecht, B. Bukau, M.P. Mayer, Pathways of allosteric regulation in Hsp70 chaperones, Nat. Commun. 6 (2015) 8308. doi:10.1038/ncomms9308.

[41] M.P. Mayer, H. Schröder, S. Rüdiger, K. Paal, T. Laufen, B. Bukau, Multistep mechanism of substrate binding determines chaperone activity of Hsp70, Nat. Struct. Biol. 7 (2000) 586-593. doi:10.1038/76819.

[42] E.B. Bertelsen, L. Chang, J.E. Gestwicki, E.R.P. Zuiderweg, Solution conformation of wild-type E. coli Hsp70 (DnaK) chaperone complexed with ADP and substrate, Proc. Natl. Acad. Sci. U.S.a. 106 (2009) 8471-8476. doi:10.1073/pnas.0903503106.

[43] W. Neupert, M. Brunner, The protein import motor of mitochondria, Nat Rev Mol Cell Biol. 3 (2002) 555-565. doi:10.1038/nrm878.

[44] B.S. Glick, Can Hsp70 proteins act as force-generating motors? Cell. 80 (1995) 11-14. doi:10.1016/0092-8674(95)90444-1.

[45] P. Goloubinoff, P. De Los Rios, The mechanism of Hsp70 chaperones: (entropic) pulling the models together, Trends Biochem. Sci. 32 (2007) 372-380. doi:10.1016/j.tibs.2007.06.008.

[46] S.M. Doyle, O. Genest, S. Wickner, Protein rescue from aggregates by powerful molecular chaperone machines, Nat Rev Mol Cell Biol. 14 (2013) 617-629. doi:10.1038/nrm3660.

[47] N. Morgner, C. Schmidt, V. Beilsten-Edmands, I.-O. Ebong, N.A. Patel, E.M. Clerico, et al., Hsp70 forms antiparallel dimers stabilized by post-translational 
modifications to position clients for transfer to Hsp90, Cell Rep. 11 (2015) 759769. doi:10.1016/j.celrep.2015.03.063.

[48] E.B. Sarbeng, Q. Liu, X. Tian, J. Yang, H. Li, J.L. Wong, et al., A functional DnaK dimer is essential for the efficient interaction with Hsp40 heat shock protein, J. Biol. Chem. 290 (2015) 8849-8862. doi:10.1074/jbc.M114.596288.

[49] A.D. Thompson, S.M. Bernard, G. Skiniotis, J.E. Gestwicki, Visualization and functional analysis of the oligomeric states of Escherichia coli heat shock protein 70 (Hsp70/DnaK), Cell Stress Chaperones. 17 (2012) 313-327. doi:10.1007/s12192-011-0307-1.

[50] C.E. Angelidis, I. Lazaridis, G.N. Pagoulatos, Aggregation of hsp70 and hsc70 in vivo is distinct and temperature- dependent and their chaperone function is directly related to non- aggregated forms, Febs J. 259 (1999) 505-512. doi:10.1046/j.1432-1327.1999.00078.x.

[51] D.E. Spratt, H. Walden, G.S. Shaw, RBR E3 ubiquitin ligases: new structures, new insights, new questions, Biochem. J. 458 (2014) 421-437. doi:10.1042/BJ20140006.

[52] L.D. D'Andrea, L. Regan, TPR proteins: the versatile helix, Trends Biochem. Sci. 28 (2003) 655-662. doi:10.1016/j.tibs.2003.10.007.

[53] E.R.G. Main, Y. Xiong, M.J. Cocco, L. D'Andrea, L. Regan, Design of stable alpha-helical arrays from an idealized TPR motif, Structure. 11 (2003) 497-508. doi:10.1016/S0969-2126(03)00076-5.

[54] A.L. Cortajarena, T. Kajander, W. Pan, M.J. Cocco, L. Regan, Protein design to understand peptide ligand recognition by tetratricopeptide repeat proteins, Protein Eng. Des. Sel. 17 (2004) 399-409. doi:10.1093/protein/gzh047.

[55] A.L. Cortajarena, L. Regan, Ligand binding by TPR domains, Protein Sci. 15 (2006) 1193-1198. doi:10.1110/ps.062092506.

[56] R.K. Allan, T. Ratajczak, Versatile TPR domains accommodate different modes of target protein recognition and function, Cell Stress Chaperones. 16 (2011) 353-367. doi:10.1007/s12192-010-0248-0.

[57] N. Zeytuni, R. Zarivach, Structural and functional discussion of the tetra-tricopeptide repeat, a protein interaction module, Structure. 20 (2012) 397-405. doi:10.1016/j.str.2012.01.006.

[58] V.A. Assimon, D.R. Southworth, J.E. Gestwicki, Specific Binding of Tetratricopeptide Repeat Proteins to Heat Shock Protein 70 (Hsp70) and Heat Shock Protein 90 (Hsp90) Is Regulated by Affinity and Phosphorylation, Biochemistry. 54 (2015) 7120-7131. doi:10.1021/acs.biochem.5b00801.

[59] J. Yang, S.M. Roe, M.J. Cliff, M.A. Williams, J.E. Ladbury, P.T.W. Cohen, et al., Molecular basis for TPR domain-mediated regulation of protein phosphatase 5, Embo J. 24 (2005) 1-10. doi:10.1038/sj.emboj.7600496.

[60] K. Lapouge, S.J. Smith, P.A. Walker, S.J. Gamblin, S.J. Smerdon, K. Rittinger, Structure of the TPR domain of p67phox in complex with Rac•GTP, Mol. Cell. 6 (2000) 899-907.

[61] Z. Xu, E. Kohli, K.I. Devlin, M. Bold, J.C. Nix, S. Misra, Interactions between the quality control ubiquitin ligase CHIP and ubiquitin conjugating enzymes, BMC Struct Biol. 8 (2008) 26-26. doi:10.1186/1472-6807-8-26. 
[62] M.B. Metzger, J.N. Pruneda, R.E. Klevit, A.M. Weissman, RING-type E3 ligases: Master manipulators of E2 ubiquitin-conjugating enzymes and ubiquitination, Biochim. Biophys. Acta. (2013). doi:10.1016/j.bbamcr.2013.05.026.

[63] D.M. Wenzel, K.E. Stoll, R.E. Klevit, E2s: structurally economical and functionally replete, Biochem. J. 433 (2011) 31-42. doi:10.1042/BJ20100985.

[64] S.E. Soss, Y. Yue, S. Dhe-Paganon, W.J. Chazin, E2 conjugating enzyme selectivity and requirements for function of the E3 ubiquitin ligase CHIP, J. Biol. Chem. 286 (2011) 21277-21286. doi:10.1074/jbc.M111.224006.

[65] S.E. Soss, K.L. Rose, S. Hill, S. Jouan, W.J. Chazin, Biochemical and Proteomic Analysis of Ubiquitination of Hsc70 and Hsp70 by the E3 Ligase CHIP, PLoS ONE. 10 (2015) e0128240. doi:10.1371/journal.pone.0128240.

[66] M.D. Ohi, C.W. Vander Kooi, J.A. Rosenberg, W.J. Chazin, K.L. Gould, Structural insights into the U-box, a domain associated with multi-ubiquitination, Nat. Struct. Biol. 10 (2003) 250-255. doi:10.1038/nsb906.

[67] S. Hatakeyama, M. Yada, M. Matsumoto, N. Ishida, K.I. Nakayama, U box proteins as a new family of ubiquitin-protein ligases, J. Biol. Chem. 276 (2001) 33111-33120. doi:10.1074/jbc.M102755200.

[68] A. Plechanovová, E.G. Jaffray, M.H. Tatham, J.H. Naismith, R.T. Hay, Structure of a RING E3 ligase and ubiquitin-loaded E2 primed for catalysis, Nature. 489 (2012) 115-120. doi:10.1038/nature11376.

[69] H. Dou, L. Buetow, G.J. Sibbet, K. Cameron, D.T. Huang, BIRC7-E2 ubiquitin conjugate structure reveals the mechanism of ubiquitin transfer by a RING dimer, Nat. Struct. Mol. Biol. 19 (2012) 876-883. doi:10.1038/nsmb.2379.

[70] H. Dou, L. Buetow, G.J. Sibbet, K. Cameron, D.T. Huang, Essentiality of a nonRING element in priming donor ubiquitin for catalysis by a monomeric E3, Nat. Struct. Mol. Biol. 20 (2013) 982-986. doi:10.1038/nsmb.2621.

[71] J.N. Pruneda, P.J. Littlefield, S.E. Soss, K.A. Nordquist, W.J. Chazin, P.S. Brzovic, et al., Structure of an E3:E2 Ub Complex Reveals an Allosteric Mechanism Shared among RING/U-box Ligases, Mol. Cell. 47 (2012) 933-942. doi:10.1016/j.molcel.2012.07.001.

[72] C. Graf, M. Stankiewicz, R. Nikolay, M.P. Mayer, Insights into the conformational dynamics of the E3 ubiquitin ligase CHIP in complex with chaperones and E2 enzymes, Biochemistry. 49 (2010) 2121-2129. doi:10.1021/bi901829f.

[73] S.-B. Qian, L. Waldron, N. Choudhary, R.E. Klevit, W.J. Chazin, C. Patterson, Engineering a ubiquitin ligase reveals conformational flexibility required for ubiquitin transfer, J. Biol. Chem. 284 (2009) 26797-26802.

doi:10.1074/jbc.M109.032334.

[74] L. Wang, Y.-T. Liu, R. Hao, L. Chen, Z. Chang, H.-R. Wang, et al., Molecular mechanism of the negative regulation of Smad1/5 protein by carboxyl terminus of Hsc70-interacting protein (CHIP), J. Biol. Chem. 286 (2011) 15883-15894. doi:10.1074/jbc.M110.201814.

[75] P. Muller, E. Ruckova, P. Halada, P.J. Coates, R. Hrstka, D.P. Lane, et al., Cterminal phosphorylation of $\mathrm{Hsp} 70$ and $\mathrm{Hsp90}$ regulates alternate binding to cochaperones CHIP and HOP to determine cellular protein folding/degradation balances, Oncogene. 32 (2013) 3101-3110. doi:10.1038/onc.2012.314. 
[76] M.V. Powers, K. Jones, C. Barillari, I. Westwood, R.L.M. van Montfort, P. Workman, Targeting HSP70: the second potentially druggable heat shock protein and molecular chaperone? Cell Cycle. 9 (2010) 1542-1550. doi:10.4161/cc.9.8.11204.

[77] M. Kajiro, R. Hirota, Y. Nakajima, K. Kawanowa, K. So-ma, I. Ito, et al., The ubiquitin ligase CHIP acts as an upstream regulator of oncogenic pathways, Nat. Cell Biol. 11 (2009) 312-319. doi:10.1038/ncb1839.

[78] I. Grummt, Life on a planet of its own: regulation of RNA polymerase I transcription in the nucleolus, Genes Dev. 17 (2003) 1691-1702. doi:10.1101/gad.1098503R.

[79] L. Kundrat, L. Regan, Identification of residues on Hsp70 and Hsp90 ubiquitinated by the cochaperone CHIP, J. Mol. Biol. 395 (2010) 587-594. doi:10.1016/j.jmb.2009.11.017.

[80] T.R. Alderson, J.H. Kim, J.L. Markley, Dynamical Structures of Hsp70 and Hsp70-Hsp40 Complexes, Structure. (2016). doi:10.1016/j.str.2016.05.011.

[81] E.R.P. Zuiderweg, Structural basis of the allosteric trigger of the Hsp70 chaperone proteins, Nature Precedings. (2008) 1-14. doi:10.1038/npre.2008.1978.1.

[82] Z.T. Young, J.N. Rauch, V.A. Assimon, U.K. Jinwal, M. Ahn, X. Li, et al., Stabilizing the Hsp70-Tau Complex Promotes Turnover in Models of Tauopathy, Cell Chem Biol. 23 (2016) 992-1001. doi:10.1016/j.chembiol.2016.04.014.

[83] A. Plechanovová, E.G. Jaffray, S.A. McMahon, K.A. Johnson, I. Navrátilová, J.H. Naismith, et al., Mechanism of ubiquitylation by dimeric RING ligase RNF4, Nat. Struct. Mol. Biol. 18 (2011) 1052-1059. doi:10.1038/nsmb.2108. 


\section{FIGURE LEGENDS}

\section{Figure 1. Structural changes in Hsp70 due to ATP hydrolysis}

(a) The structure of ATP-bound Hsp70 features tight packing of the $\beta$-substrate binding domain ( $\beta$-SBD) against the nucleotide binding domain (NBD) and the helical lid domain swung out away from $\beta$-SBD. Structure shown is the Hsp70 homolog DnaK in the ATPbound state (PDB ID: 4B9Q). (b) The structure of ADP-bound Hsp70 features disengagement of the $\beta$-SBD from the NBD and clamping of the helical lid domain onto the $\beta$-SBD in a conformation that tightly binds misfolded clients. Structure shown is the Hsp70 homolog DnaK in the ADP-bound state (PDB ID: 2KHO).

\section{Figure 2. The Hsp70 protein refolding cycle.}

The refolding cycle begins with ATP binding to the nucleotide binding domain (NBD) of Hsp70, followed by binding of HSP40 and a misfolded client to Hsp70. Binding of the client and HSP40 stimulate ATP hydrolysis, altering the conformation of Hsp70. HSP40 dissociates from Hsp70 and a nucleotide exchange factor (NEF) is recruited to the Hsp70 NBD. Interaction with the NEF triggers dissociation of the nucleotide from the Hsp70 NBD, which is followed by release of the client and dissociation of the NEF. This effectively regenerates Hsp70 in a form competent for binding ATP and repeating the cycle. The dissociated client is either refolded, or remains misfolded and can be brought into the pathway again for further rounds of refolding.

Figure 3. General scheme of the ubiquitination cascade.

All ubiquitination cascades begin with ATP-dependent modification of the active site cysteine from an E1 enzyme (b/ue) by ubiquitin (green), followed by transfer of the ubiquitin to the active site cysteine of an E2 enzyme (purple). U-box and RING-type E3 enzymes (grey) catalyze direct transfer of a ubiquitin molecule from an E2 ubiquitin conjugate to a lysine residue. HECT and RING-between-RING-type E3 ligases (brown) first catalyze transfer of a ubiquitin molecule from an E2 ubiquitin conjugate to a cysteine on the HECT and RING-between-RING-type E3 ligase, followed by transfer to a lysine residue. Repeated interaction of E2 ubiquitin conjugates with an E3 ubiquitin ligase results in polyubiquitination.

\section{Figure 4. Domain organization of CHIP and interactions with an E2 ubiquitin conjugate.}

(a) Structure of a CHIP dimer shown as cartoons and a translucent surface. The second protomer of the dimer is shown as a ghost with reduced opacity. U-box, TPR, and linker domains are indicated by text. (b) Structure of a CHIP dimer in complex with an E2 ubiquitin conjugate, again the second protomer of the CHIP dimer is shown as a ghost. 
Figure 5. Asymmetric and symmetric dimer models for CHIP.

Block representation of asymmetric and symmetric dimers of CHIP (cyan, and green). Lines indicate range of motion for domains moving between the asymmetric and symmetric forms.

Figure 6. Structure of CHIP-TPR and interactions with constructs containing IEEVD motifs.

(a) Structure of CHIP-TPR (white, PDB ID: 2C2L) superimposed with a single protomer of the full-length CHIP dimer (translucent white, PDB ID: 2C2L). CHIP-TPR helices h1 (red), h2 (orange), h3 (yellow), h4 (green), h5 (cyan), h6 (blue), and h7 (purple) are highlighted. Helix $\mathrm{h} 7$ is extended in full-length CHIP to form one of the two helices of the linker domain. (b) Structure of CHIP-TPR (orange, PDB ID: 3Q49) in complex with the C-terminal EEVD motif of Hsp70 (cyan, PDB ID: 3Q49). (c) Structure of CHIP-TPR (blue, PDB ID: 2C2L) in complex with the C-terminal EEVD motif of HSP90 (pink, PDB ID: 2C2L). (d) Structure of CHIP-TPR (purple, PDB ID: 4KBQ) in complex with the Hsc70-lid-Tail domain (yellow, PDB ID: 4KBQ), including the EEVD motif.

Figure 7. Models of the CHIP:Hsp70 chaperoned ubiquitination complex.

(a) A model of the CHIP:Hsp70:E2 ubiquitin complex for ADP-bound Hsp70 (PDB ID: $2 \mathrm{KHO}$ ) is shown as the molecular surfaces with the CHIP dimer (grey and white, PDB ID: 2C2L), E2 (PDB ID: 3TGD), ubiquitin (green, PDB ID: 1UBQ), and Hsp70 (blue, PDB ID: 2KHO). The CHIP protomer show in white binds the E2 ubiquitin and Hsp70, while the CHIP protomer in grey shares a binding interface for ubiquitin. The modeled structure of the E2 ubiquitin conjugate bound to CHIP is based upon a recent RINGbound E2 ubiquitin conjugate structure (PDB ID: 4AP4)[83]. (b) A model of the CHIP:Hsp70:E2 ubiquitin complex for ATP-bound Hsp70 (PDB ID: 4B9Q) colored as described in (a).

\section{GRAPHICAL ABSTRACT}

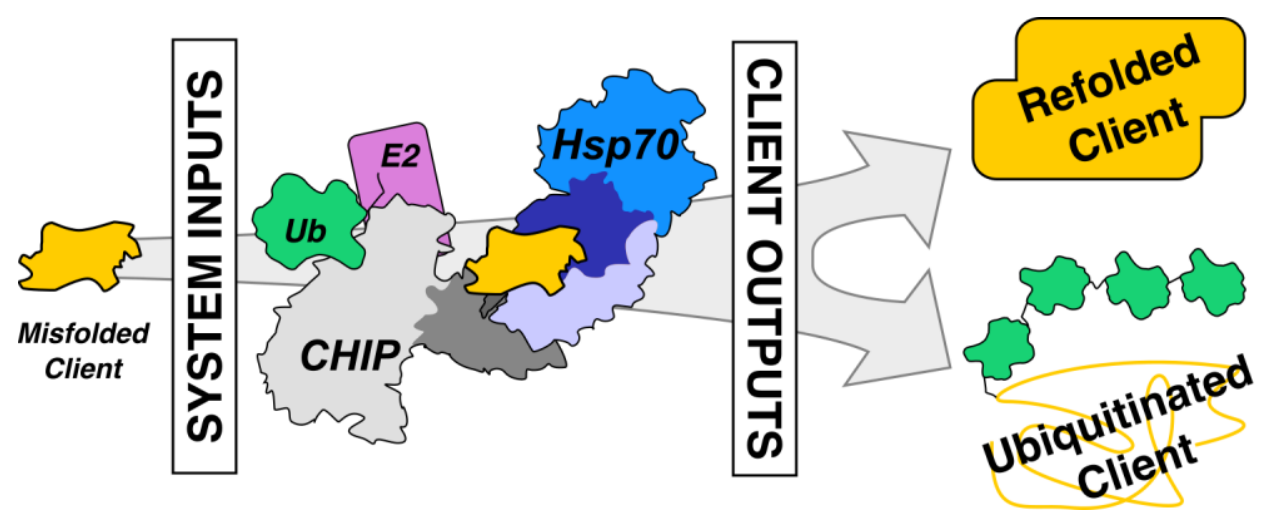


a

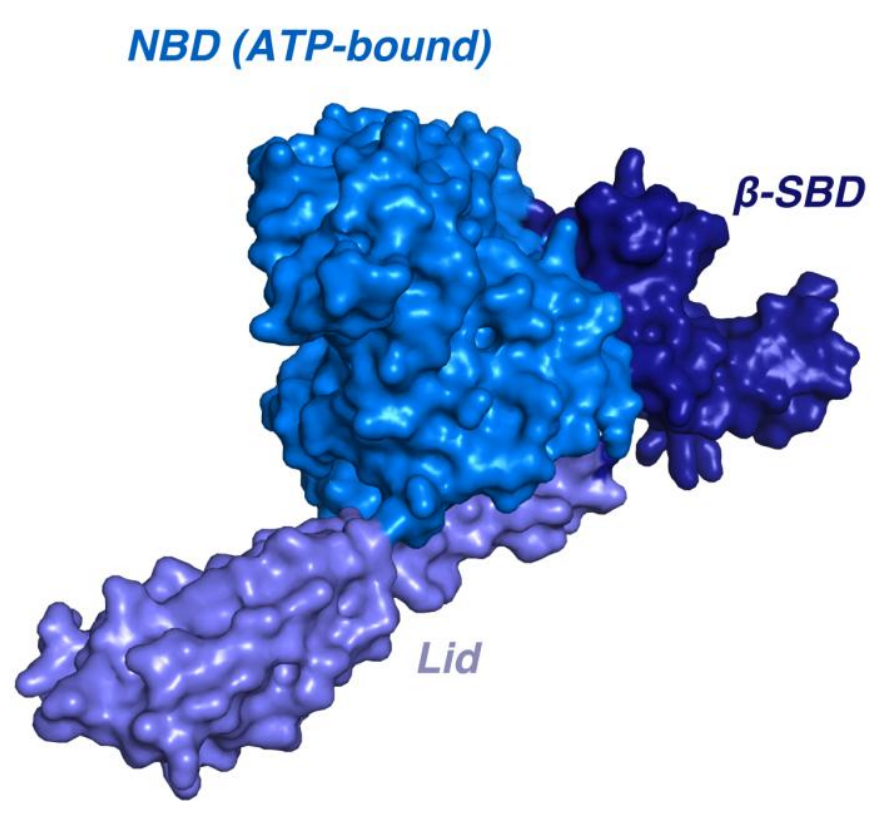

b

NBD (ADP-bound)

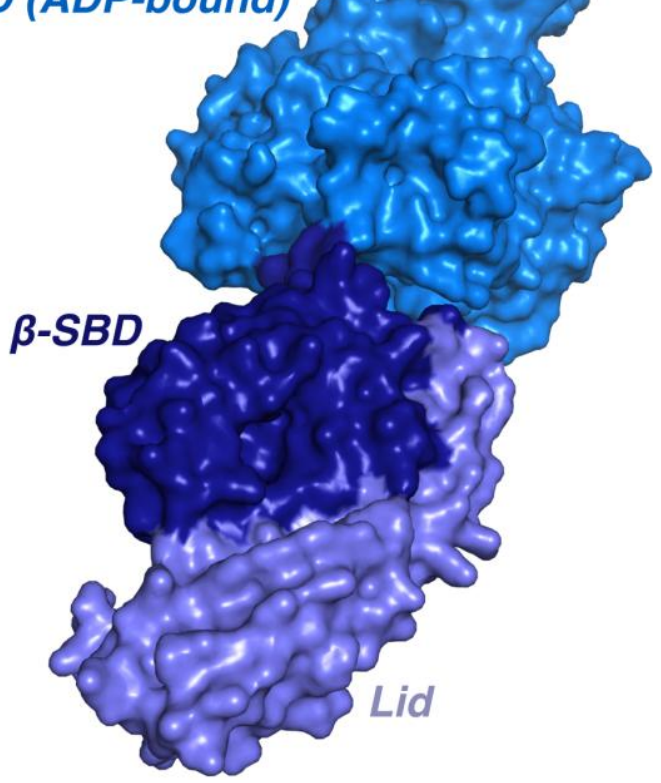




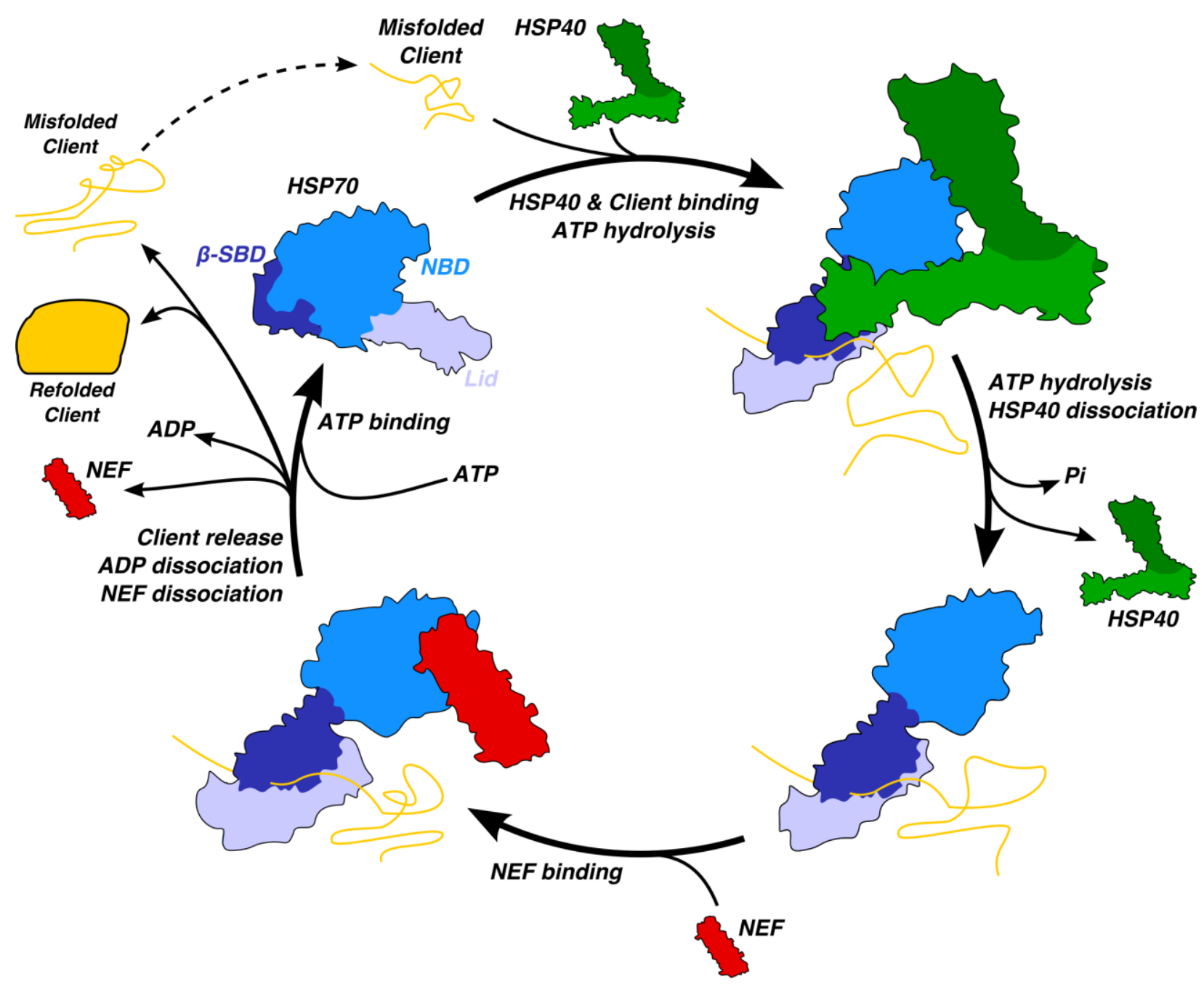




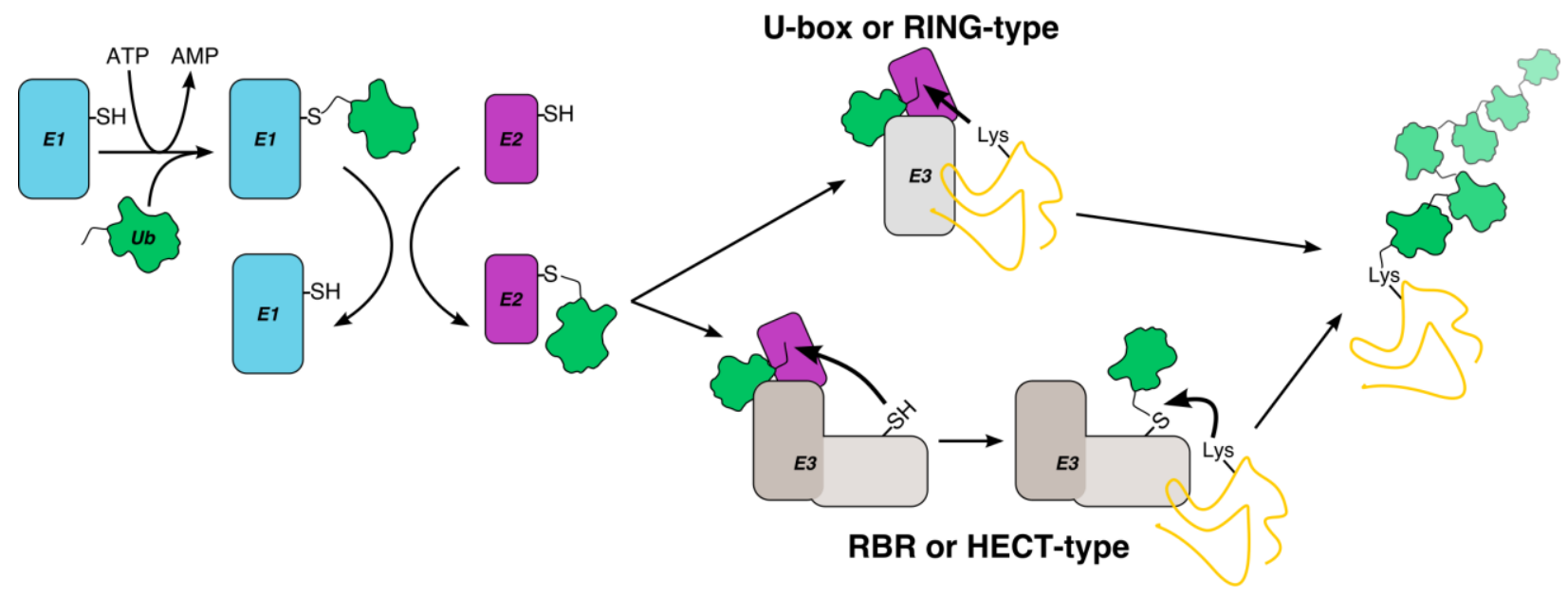


a

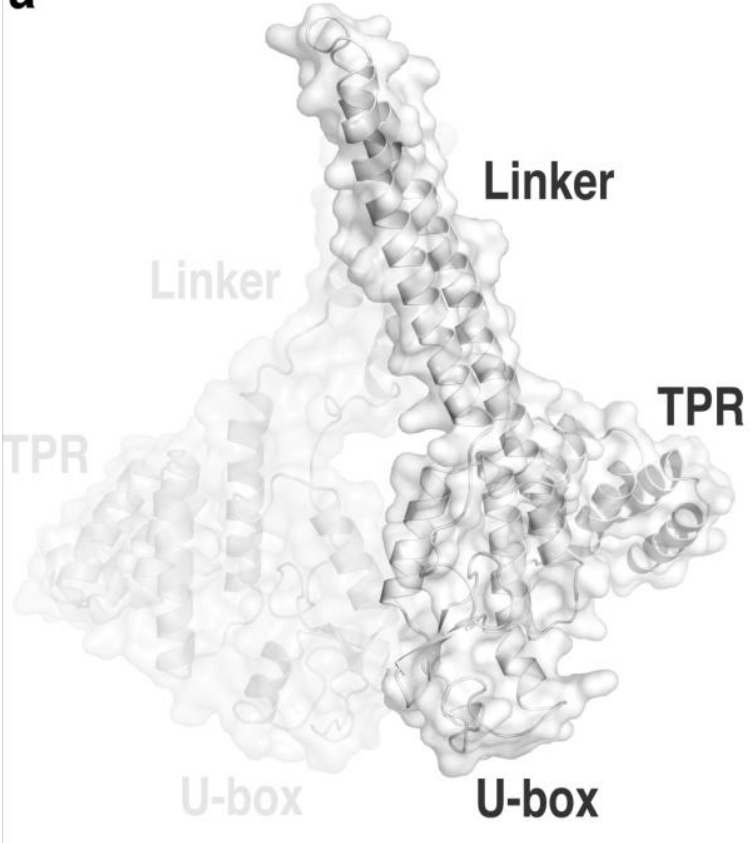

b

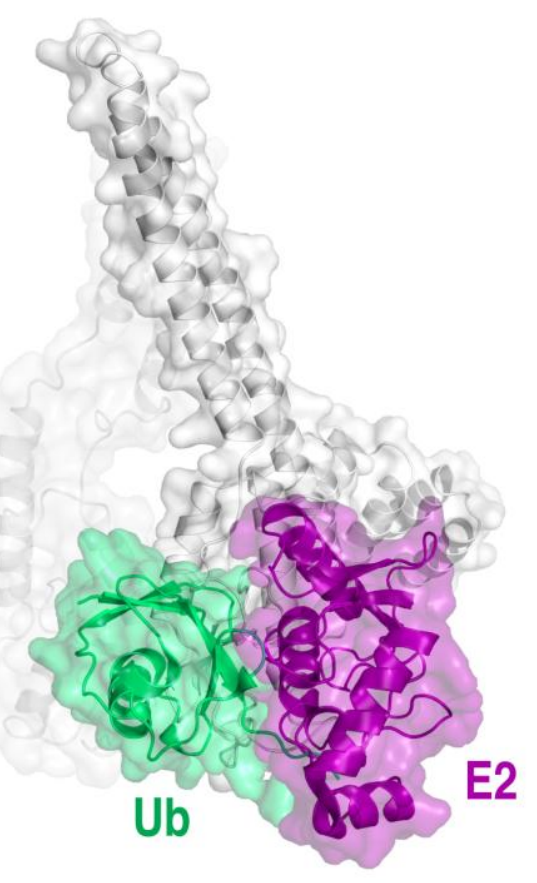




\section{Asymmetric}

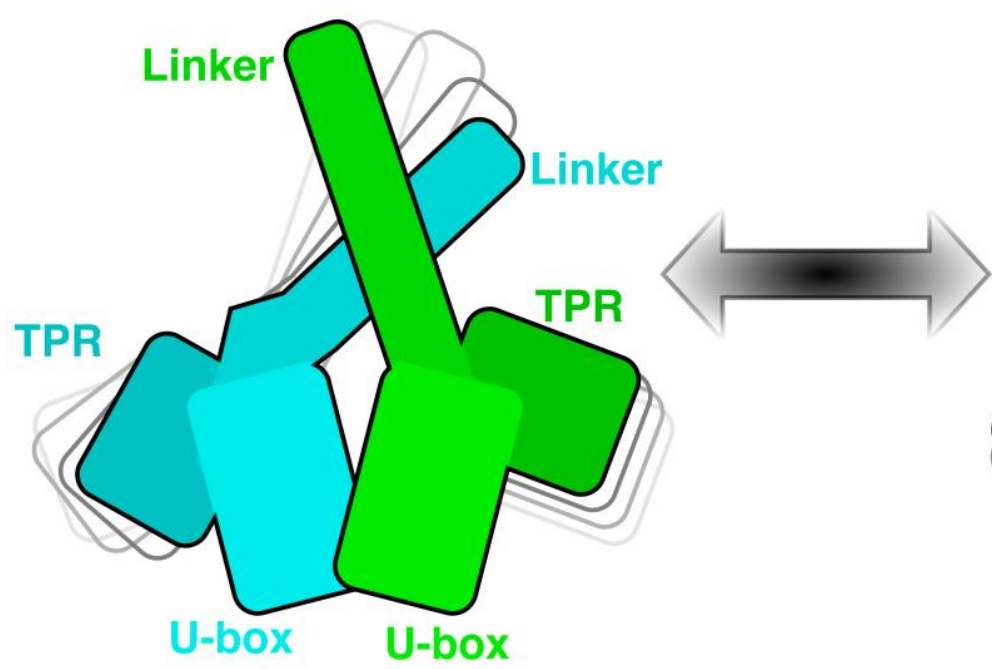

Symmetric

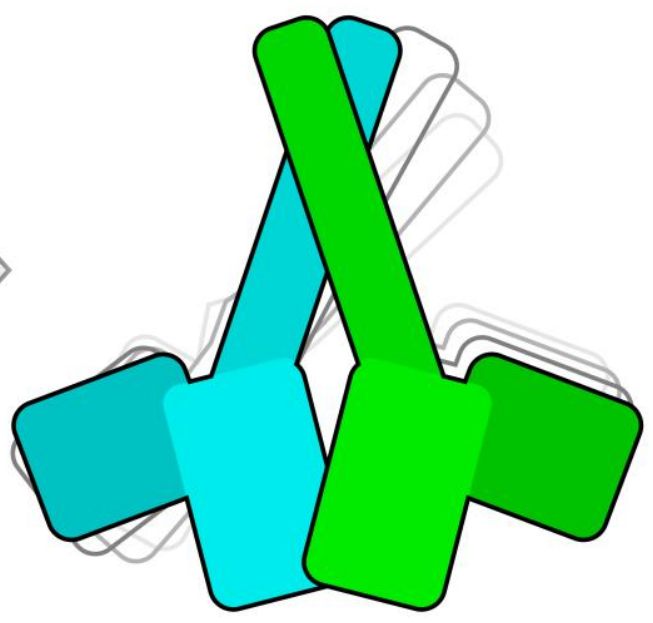


a

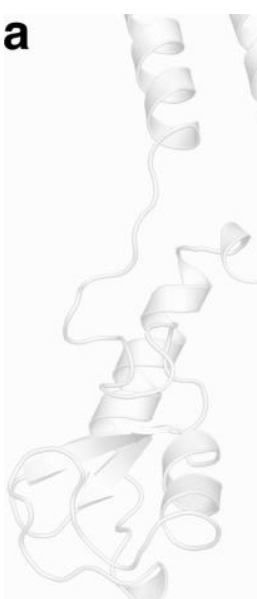

C

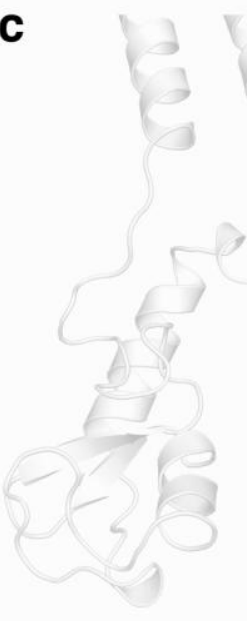

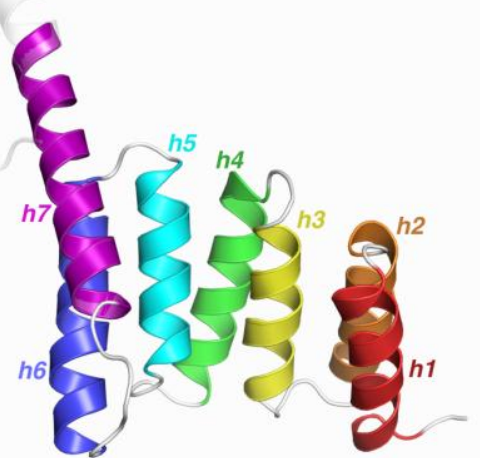

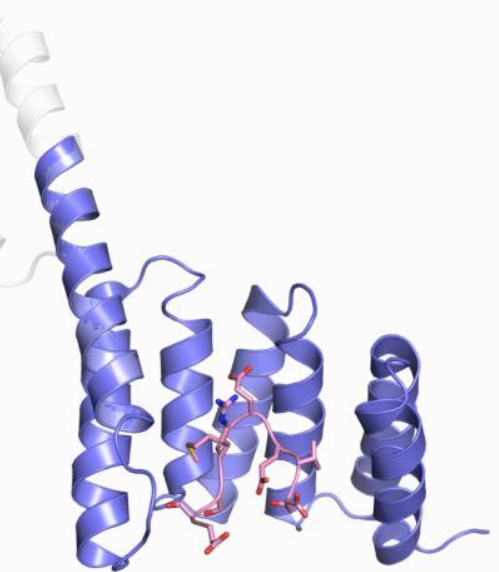

b

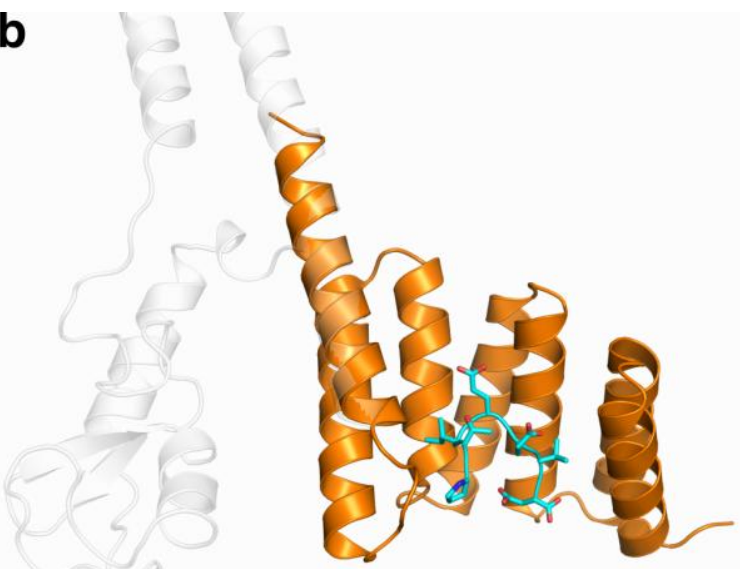

d

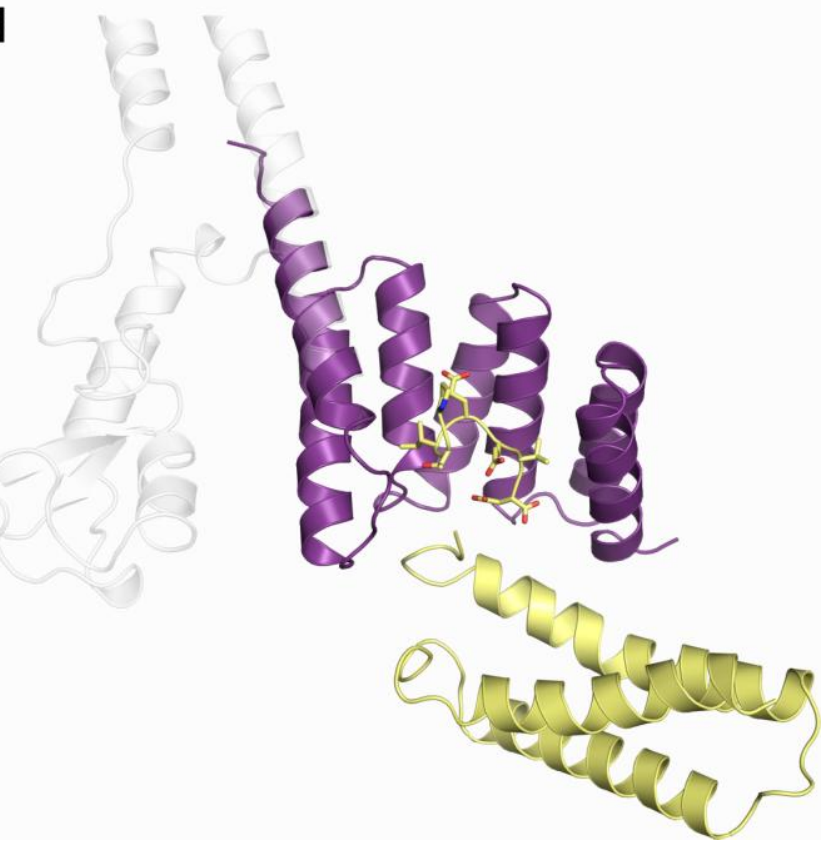




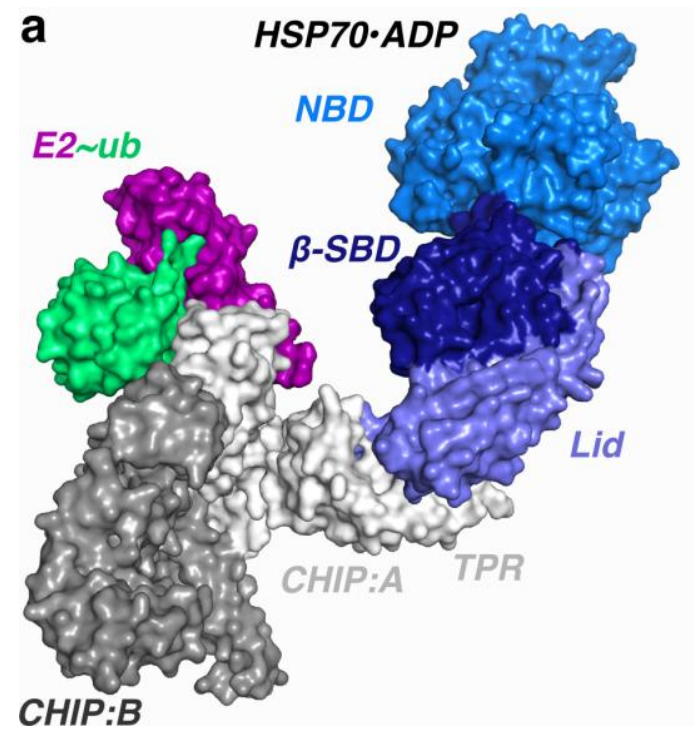

b

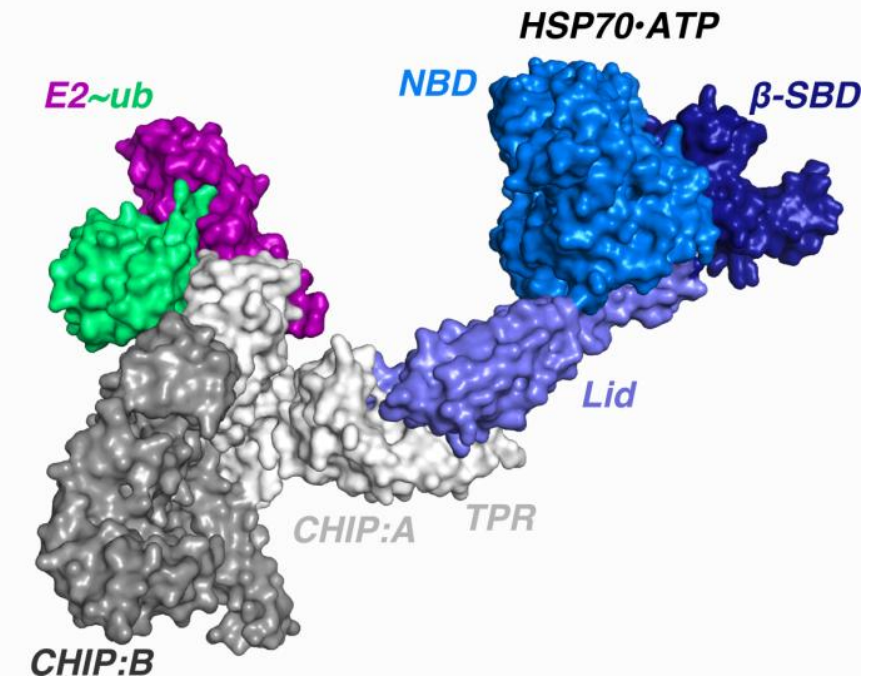

